Int. J. Morphol.,

35(2):765-775, 2017

\title{
Primeros Ramos del Nervio Plantar Lateral Revisión Anátomo - Clínica
}

\author{
First Branches of the Lateral Plantar Nerve. \\ Anatomo- Clinical Review
}

\author{
Mariano del Sol ${ }^{1}$ \& Bélgica Vásquez ${ }^{2}$
}

DEL SOL, M. \& VÁSQUEZ, B. Primeros ramos del nervio plantar lateral. Revisión anátomo-clínica. Int. J. Morphol., 35(2):765$775,2017$.

RESUMEN: El nervio plantar lateral es un ramo terminal del nervio tibial, inerva la mayor parte de la musculatura del pie, y otorga inervación sensitiva a parte de la planta del pie. En esta revisión abordamos diversos aspectos del nervio plantar lateral desde su origen hasta su división, dando énfasis a aquellos ramos que por su disposición anatómica han sido señalados como causantes de síndrome de compresión, y a variaciones anatómicas importantes que deben ser conocidas cuando se aborda la región plantar. Numerosos estudios se han realizado para describir el trayecto y relaciones de los primeros ramos del nervio plantar lateral. Los ramos emitidos directamente por el nervio plantar lateral están destinados a los músculos abductor del dedo mínimo y cuadrado plantar, además de un ramo cutáneo lateral y ramos vasculares para los vasos plantares laterales y para el ligamento plantar largo. Como variación anatómica es posible encontrar ramos calcáneos mediales, tronco común de ramos calcáneo medial y para el músculo abductor del dedo mínimo, 2 ó 3 ramos para el músculo cuadrado plantar, tronco común para los músculos abductor del dedo mínimo y cuadrado plantar, ramo para el músculo flexor corto del dedo mínimo y ramo "anastomótico" para el nervio plantar medial. Cada vez más, es más necesario tener conocimientos acabados de la anatomía del pie, particularmente en aquellos procedimientos quirúrgicos que requieren precisión, con abordajes menos radicales y buenos resultados.

PALABRAS CLAVE: Nervio plantar lateral; Anatomía; Síndrome de compresión.

\section{INTRODUCCIÓN}

La inervación del pie se realiza a través de ramos de los nervios fibular superficial, fibular profundo y tibial y, principalmente, sus ramos de división, los nervios plantares lateral y medial, quienes inervan el mayor territorio del pie, tanto muscular como cutáneo.

Ramos originados del nervio plantar lateral, en especial los más proximales, han sido mencionados en las últimas décadas como eventuales causantes de dolor subcalcáneo, debido al síndrome de compresión de uno de ellos. A pesar que el síndrome del túnel tarsiano es menos frecuente que el síndrome del túnel carpiano, sus efectos pueden ser bastante graves debido a que comprometen la marcha y la posición de bipedestación, afectando seriamente la calidad de vida del paciente. Se sabe que gran parte de los ramos nerviosos que pueden ser afectados por compre- sión, son principalmente los ramos terminales del nervio tibial (ramos calcáneos mediales) o los primeros ramos del nervio plantar lateral (variación de origen de los ramos calcáneos mediales y ramo para el músculo abductor del dedo mínimo) quienes se ven especialmente comprometidos en la región calcánea y parte posterior de la planta del pie.

Debido a lo anterior, numerosos estudios se han realizado para describir de manera precisa el trayecto y relaciones de estos primeros ramos del nervio plantar lateral. Existen estudios como los de Presley et al. (2012) cuyo objetivo principal de su investigación fue documentar la capacidad del ultrasonido de alta resolución para identificar con precisión el primer ramo del nervio plantar lateral, utilizando para ello inyecciones perineurales guiadas por ecografía en un modelo cadavérico.

\footnotetext{
${ }^{1}$ Centro de Excelencia en Estudios Morfológicos y Quirúrgicos (CEMyQ), Universidad de La Frontera, Chile.

${ }^{2}$ Facultad de Ciencias de la Salud, Universidad de Tarapacá, Chile.
} 
Origen del nervio plantar lateral. El nervio plantar lateral solo en su origen se relaciona con el nervio plantar medial, siendo más vertical que éste, se dirige distalmente relacionado con la cara medial del calcáneo, cubierto por los vasos tibiales posteriores hasta el margen superior del músculo abductor del hallux, donde la arteria tibial posterior se divide en los arterias plantares medial y lateral, acompañadas de sus respectivas venas. Se coloca en contacto con la cara superficial de la cabeza medial del músculo cuadrado plantar, más proximalmente cuando más alto es el origen de esta cabeza en el calcáneo. De esta manera, la cabeza medial del músculo cuadrado plantar separa la cara medial del calcáneo del nervio plantar lateral y de sus ramos más proximales (Fig. 1).

El nervio plantar lateral describe una ligera curva, cóncava en sentido anterosuperior, se dirige inferior y anteriormente, relacionándose al inicio directamente con las arterias plantares, luego se coloca en relación con la cara pro-

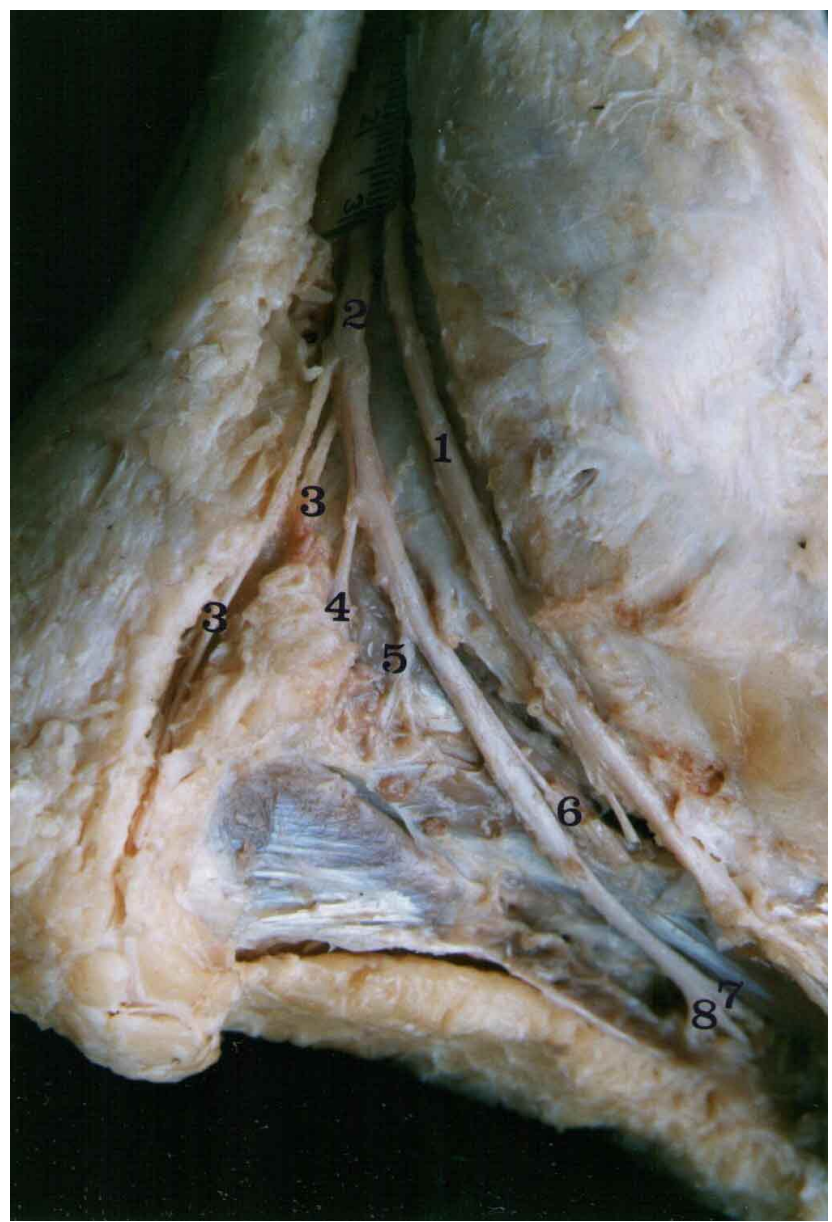

Fig. 1. Vista inferomedial del pie mostrando los nervios plantares. Nervio plantar medial; 2. Nervio plantar lateral; 3. Ramos calcáneos mediales; 4. Ramo para el músculo abductor del dedo mínimo; 5. Ramo posterior para el músculo cuadrao plantar; 6. Ramo anterior para el músculo cuadrado plantar; 7. Ramo profundo del nervio plantar lateral; 8. Ramo superficial del nervio plantar lateral. funda del músculo abductor del hallux, del cual se encuentra separado por las numerosas anastomosis que se establecen entre las venas plantares laterales, que formarán posteriormente las venas tibiales posteriores, a nivel del margen superior del músculo abductor del hallux (Figs. 2 y 3 ).

El nervio plantar lateral cruza en forma de $\mathrm{X}$ alargada al músculo abductor del hallux pasando al compartimiento intermedio del pie a través de un foramen formado a nivel del margen inferior del referido músculo. En este lugar se torna horizontal y adquiere una forma aplanada, luego se ubica entre los músculos flexor corto de los dedos, inferiormente y cuadrado plantar, superiormente, cruzando a este último músculo en forma de una $\mathrm{X}$ alargada. En seguida avanza en sentido lateral y anterior paralelo al tendón del músculo flexor largo de los dedos y termina dividiéndose a nivel del margen lateral de la cabeza lateral del músculo cuadrado plantar.

La arteria tibial posterior se divide normalmente, a nivel del margen superior del músculo abductor del hallux, originándose por tanto, al mismo nivel de las venas tibiales posteriores, producto de reunión de las venas plantares mediales y laterales, las cuales también, contribuyen a separar dicho nervio de la cara profunda del músculo abductor del hallux (Figs. 2 y 3 ).

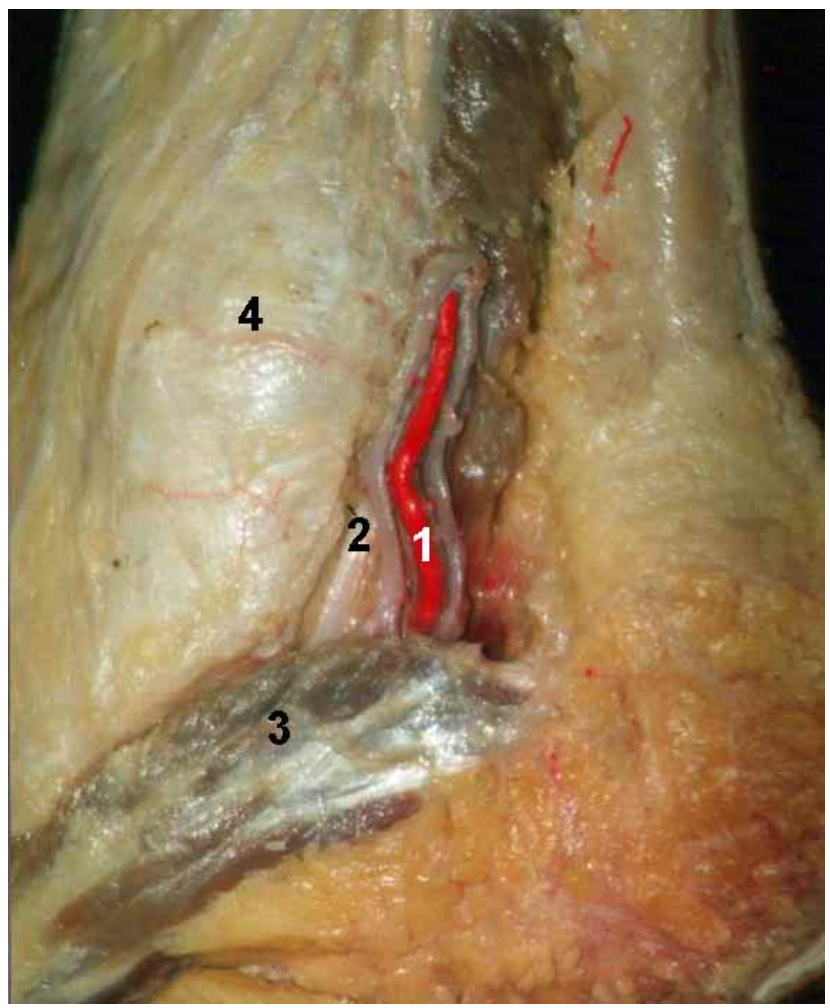

Fig. 2. Vista medial del pie. 1. Arteria tibial posterior; 2. Nervio plantar medial; 3. Músculo abductor del hálux; 4. Maléolo medial. 
El origen conjunto del nervio plantar lateral con ramos calcáneos mediales ha sido señalado entre otros por Kopell \& Thompson (1960), Henricson \& Westlin (1984), Dellon \& Mackinnon (1984), Belen (1985), y del Sol et al. (1994), constituyendo por su bajo porcentaje de ocurrencia una variación anatómica.

Por otra parte, el origen conjunto del nervio plantar lateral con el ramo para el músculo abductor del dedo mínimo fue observado por del Sol \& Prates (1988) y del Sol et al. (2002), situación semejante a lo que habían señalado Baxter \& Thigpen (1984), para quienes el nervio tibial se divide en cuatro o cinco ramos, siendo uno o dos superficiales conocidos como ramos mediales y los otros profundos. Sin embargo, estos mismos autores indicaron que exactamente a distal del ramo calcáneo medial, el nervio tibial se divide en los dos nervios plantares y el ramo para el músculo abductor del dedo mínimo.

De acuerdo a los señalado por Belen, Baxter \& Thigpen y del Sol (2002), las situaciones planteadas no son más que una excepción a la normalidad, en consecuencia pueden ser consideradas un tipo de variación anatómica. Se debe tener en consideración que, un origen alto del ramo para el músculo abductor del dedo mínimo, debiera presentar un trayecto más vertical y medial al calcáneo para posteriormente rodear anteriormente a la tuberosidad calcánea.

El nervio plantar lateral se origina proximal al margen superior del músculo abductor del hallux (del Sol et al., 1995). Sin embargo se ha indicado que este nervio se origina cubierto por el músculo abductor del hallux (Tandler, 1933), o cubierto por el origen del referido músculo (Schaeffer, 1943). Más difícil es encontrar un origen inferior al músculo abductor del hallux (Bourgery (1844) y Woodburne (1984). Esta última situación nos parece poco probable, basta observar que cuando el nervio plantar lateral alcanza el margen superior del músculo abductor del hallux se encuentra notablemente separado del nervio plantar medial e incluso ha otorgado en la mayoría de los casos su primer ramo mixto (del Sol et al., 1991). Otro punto señalado para el origen del nervio plantar lateral lo constituye el retináculo de los músculos flexores ya sea cuando lo cruza, a nivel del mismo, profundo a éste o a nivel de su margen inferior.

\section{Ramos del nervio plantar lateral desde su origen hasta su división}

Hay que destacar, que son pocos los trabajos específicos sobre cada uno de los ramos que proporciona el nervio plantar lateral antes de su división, reportados en la literatura especializada. Los ramos emitidos directamente por el nervio plantar lateral de manera normal, son los ramos para el músculo abductor del dedo mínimo y cuadrado plantar, además de un ramo cutáneo, denominado por del Sol \& Prates (1990) ramo cutáneo lateral.

En diversos artículos se han descrito otros ramos del nervio plantar lateral antes de su división en ramos superficial y profundo. Así es posible encontrar ramos calcáneos mediales (del Sol \& Prates, 1994) tronco común de donde emergen los ramos calcáneo medial y para el músculo abductor del dedo mínimo, ramos vasculares para los vasos plantares, ramo(s) para el músculo cuadrado plantar (del Sol \& Prates, 1994), tronco común para los músculos abductor del dedo mínimo y cuadrado plantar, ramo cutáneo lateral, ramo para el músculo flexor corto del dedo mínimo, ramo "anastomótico" para el nervio plantar medial y ramo para el ligamento plantar largo y ligamento calcáneocuboideo plantar, entre otros.

Es importante tener en consideración el orden de enumeración de los ramos emitidos por el nervio plantar lateral antes de su división, principalmente porque proporciona un orden lógico de la secuencia en que se originan de proximal a distal estos ramos. Esto último, resulta ser un elemento clave para el anatomista y el cirujano para determinar con seguridad y certeza el origen de dichos ramos nerviosos en un abordaje anatómico o quirúrgico.

Prácticamente, no se hace mención en los artículos consultados a ramos cutáneos que se originen del nervio plantar lateral, ya sea porque no se ha otorgado la importancia adecuada, o porque efectivamente resultó difícil observar un ramo cutáneo emergiendo en esta parte del nervio.

El origen de ramos calcáneos mediales ya ha sido comentada extensamente por Soibelman en su tesis titulada «Observaciones anatómicas sobre los ramos calcáneos mediales del nervio tibial en el Hombre» y por del Sol (1992a y 1994) para quienes, estos ramos, en la mayoría de los casos, se originan exclusiva y directamente del nervio tibial antes de su bifurcación, mencionando como menos común un origen de ramos múltiples originándose del nervio tibial y de uno de los nervios plantares, siendo así extremadamente raro el origen de ramos calcáneos mediales, exclusivamente de los nervios plantares. En relación a lo mismo, Martin-Oliva et al. (2013) reportaron que en el $25 \%$ de los casos los ramos calcáneos mediales emergen del nervio plantar lateral. Por otra parte, Ulcay et al. (2014), reportaron en un estudio en fetos al nervio calcáneo medial originándose bilateralmente en el 16,7 \% de los casos. Resultados muy dispares de los observados por Torres \& Ferreira (2012), quienes en 50 disecciones solo en un caso ( $2 \%$ ) encontaron un ramo calcáneo medial originándose del nervio plantar lateral y en 2 ca- 
sos $(4 \%)$ a ramos calcáneos mediales originándose tanto del nervio tibial, como del nervio plantar lateral.

Investigaciones realizadas por Lumsden et al. (2003). sobre la topografía distal del nervio tibial y de sus ramos, determinaron numerosas variaciones en cuanto a localización y trayecto de los ramos calcáneos mediales, hecho ya señalado por Soibelman, (1964) y del Sol \& Prates (1990).

Probablemente, el hecho de que uno o varios ramos calcáneos mediales se origine(n) del nervio plantar lateral se debe a una división precoz del nervio tibial. Sin embargo, es más probable un origen de estos ramos calcáneos mediales desde el nervio plantar medial en una división alta del nervio tibial. En cualquiera de los dos casos estos ramos cruzan el origen del músculo abductor del hallux en el proceso medial de la tuberosidad del calcáneo (Fig. 1).

Debido a lo anterior, y ante la existencia del espolón del calcáneo o la inflamación aséptica de la bolsa serosa subcalcánea que rodea al mismo, este ramo medial, puede eventualmente, ser el conductor del dolor regional. Henricson \& Westlin ya habían señalado que la división anterior del ramo calcáneo medial se encuentra interpuesto entre la fascia profunda de la parte proximal del músculo abductor del hallux y el ápice anteromedial de la tuberosidad calcánea, tornándose a ese nivel vulnerable debido a que la fascia del referido músculo es inelástica.

Es importante señalar que este trayecto inferior y anterior al proceso medial de la tuberosidad del calcáneo, más frecuentemente, según nuestra opinión, podría comprometer al ramo para el músculo abductor del dedo mínimo, el cual se encuentra en relación inmediata con esta estructura ósea, hecho ya indicado por Przylucki \& Jones (1981), Baxter $\&$ Thigpen y del Sol et al. (2002) (Fig. 1).

Probablemente los ramos anteriormente mencionados correspondan a los ramos anteriores más profundos de un ramo calcáneo medial, tal vez originado del nervio plantar lateral, dato ya reportado por Tanz (1963), quien señaló que este ramo se encuentra cubierto por la fascia y por un pequeño conjunto vascular en el punto donde estos ramos giran lateralmente, para después cruzar inferiormente, y colocarse justo en el ápice del proceso medial de la tuberosidad calcánea, siendo vulnerable a la presión del espolón calcáneo, irritación local, trauma u otra inflamación, edema o ingurgitamiento venoso, constituyéndose en una causa común de talalgia plantar.

En relación a lo mismo, del Sol \& Prates (1988) indicaron que es muy probable que el nervio señalado por Tanz pueda corresponder a una de las divisiones del ramo para el músculo abductor del dedo mínimo. De cualquier manera, algunos autores como Ribet (1953); Soilbelman; Mesdagh et al. (1980) y del Sol et al. (1988 y 2002 han señalando que la presencia de esos ramos calcáneos mediales provenientes del nervio plantar lateral constituye más bien una variación anatómica, ya que como es bien conocido, normalmente es el nervio tibial a nivel de su terminación, quien emite estos ramos, los cuales se originan habitualmente, posterior el maléolo medial a nivel del ápice del mencionado maléolo. Si el ramo calcáneo medial, como variación anatómica, tuviese su origen en el nervio plantar lateral, su trayecto sería vertical hacia abajo y ligeramente a posterior, perforaría la fascia superficial y se dividiría en dos o más ramos que seguirían la cara medial de la región calcánea, reflejándose por su cara inferior y distribuyéndose en la piel del talón.

Uno de los ramos del nervio plantar lateral, antes de su división, que más ha sido mencionado en la literatura, lo constituye, sin lugar a dudas, el ramo para el músculo abductor del dedo mínimo (mencionado por Baxter y eponinimizado por investigadores recientes). Como lo mencionaron del Sol \& Prates (1988) y del Sol et al. (2002), este ramo se origina en casi el $90 \%$ del nervio plantar lateral y ha sido indicado como uno de los causantes de dolor subcalcáneo, ya que puede ser eventualmente comprimido o estirado, ante la existencia del espolón calcáneo, causando un síndrome de compresión. Llama la atención de este nervio su grosor, que es de mayor diámetro que los otros ramos del nervio plantar lateral emitidos antes de su división. Su origen está en la parte proximal del nervio plantar lateral, por sobre o proximal al margen superior del músculo abductor del hallux (Figs. 1, 4 y 5).

A diferencia de lo relatado por Baxter \& Thigpen, para quienes el ramo para el músculo abductor del dedo mínimo es un ramo mixto, que se origina directamente del nervio tibial, al dividirse este último en cuatro o cinco ramos terminales posterior y distal al maléolo medial, para autores como del Sol \& Prates (1988), del Sol et al. (2002) y la mayoría de los investigadores consultados, como además se observa en los libros especializados de anatomía y cirugía, no existe otra versión que la terminación del nervio tibial en los nervios plantares, medial y lateral.

Lo que ocurre, como ha sido señalado anteriormente, el origen del ramo para el músculo abductor del dedo mínimo es próximo al origen del nervio plantar lateral lo que ha llevado a Baxter \& Thigpen a indicar a este ramo con un origen directo del nervio tibial, junto a los nervios plantares y calcáneos mediales.

Prácticamente todos los textos de anatomía normal, descriptiva o topográfica parecen no haberle dado impor- 
tancia al primer ramo del nervio plantar lateral (generalmente el ramo para el músculo abductor del dedo mínimo). De hecho si se examinan los libros del área y se revisan sus esquemas, se podrá constatar que este ramo ha sido representado con un origen diverso, ya sea próximo al origen del nervio plantar lateral, distante del mismo e incluso emergiendo un poco antes de su división o a una distancia considerable de la tuberosidad del calcáneo. Un origen del ramo para el músculo abductor del dedo mínimo en un tronco común con el ramo para el músculo cuadrado plantar ha sido descrito por del Sol \& Prates (1988), constituyendo una variación anatómica rara de origen de este ramo.

Este primer ramo del nervio plantar lateral (considerando variaciones a los ramos calcáneos mediales originados del nervio plantar lateral) ha sido denominado también ramo calcáneo profundo, ramo calcáneo inferior y también con el epónimo de ramo de Baxter. Señalado por muchos como causante de dolor subcalcáneo por compresión del mismo, es un ramo mixto, que inerva no solo a las dos cabezas del músculo abductor del dedo mínimo, sino también el periostio de la parte anteromedial del calcáneo y el ligamento plantar largo. Esta inervación fue descrita por del Sol \& Prates (1988 y 2002), Davis \& Schon (1995) y Louisia \& Masquelet (1999).

Autores clásicos como Testut \& Latarjet (1969) señalaron que uno de los dos ramos colaterales que da el nervio plantar lateral antes de su división en ramos superficial y profundo está destinado a los músculos flexor corto del dedo mínimo y abductor del dedo mínimo. Como lo reportaron del Sol \& Prates (1988) esta situación es incomún y constituiría una variación anatómica. Un relato similar fue expuesto por Przylucki \& Jones, quienes señalaron el origen de un ramo del nervio plantar lateral, el cual se dividía en dos ramos menores, uno de ellos inervaba al músculo flexor corto del dedo mínimo y el otro al músculo abductor del dedo mínimo, constituyéndose también en una variación anatómica. A pesar que estos autores indicaron haberla encontrado en los cuatro miembros amputados que utilizaron en el estudio. Tal vez estos autores, durante la desinserción del músculo flexor corto de los dedos en su origen calcáneo, seccionaron también la cabeza profunda del músculo abductor del dedo mínimo, en consecuencia visualizaron el ramo posterior de división del nervio para este músculo y así haber sido confundido con la inervación del músculo flexor corto de los dedos. Para que pueda ocurrir una situación como la señalada, ese ramo debería atravesar de profundidad a superficie la cabeza profunda del músculo abductor del dedo mínimo, al encontrarse ella interpuesta entre la cabeza lateral del músculo cuadrado plantar, originada en el proceso lateral de la tuberosidad calcánea y el músculo flexor corto de los dedos.
Por el trayecto y por su origen proximal, el ramo para el músculo abductor del dedo mínimo llega a la planta del pie independiente del nervio plantar lateral (Bordelon, 1983; Baxter \& Thigpen y del Sol \& Prates, 1988). Respecto a su sitio de penetración en el músculo, no existe uniformidad en los esquemas anatómicos de los diferentes textos de la especialidad. Sin embargo, lo más probable es que ingrese por el margen medial del músculo o próximo a él, por su cara profunda.

Se sabe que el ramo para el músculo abductor del dedo mínimo alcanza el margen superior del músculo abductor del hallux, cubierto por las venas tibiales posteriores (Fig. 2) y luego cruza la cara profunda del músculo a nivel de su origen, siendo separado de éste por las diversas anastomosis de las venas plantares (Przylucki \& Jones y del Sol et al., 1995) (Fig. 3). Se relaciona con la cara medial del hueso calcáneo y a seguir con la cabeza medial del músculo cuadrado plantar, rodea el proceso medial de la tuberosidad calcánea a una distancia entre 0,4 a $14 \mathrm{~mm}$. Cabe destacar que, es a este nivel donde el nervio se encuentra más expuesto a compresión, o estiramiento ante la presencia de un espolón calcáneo (Baxter \& Thigpen).

Debido a que el origen de la cabeza profunda del músculo abductor del dedo mínimo está en el proceso medial de la tuberosidad calcánea, el ramo nervioso destinado al músculo, en especial el anterior para la cabeza superficial, no cruza directamente el margen inferior inelástico del músculo abductor del hallux, sino que se encuentra cubierto. En consecuencia, es la cabeza profunda la cual se relaciona a este nivel con la fascia profunda y el margen inferior del músculo abductor del hallux (del Sol et al., 2002) Figs. 3, 4 y 5.

Por otra parte, Baxter \& Thigpen describieron una situación opuesta ya que señalaron que el trayecto ulterior del ramo para el músculo abductor del dedo mínimo cruzaba inferiormente el margen inferior del músculo abductor del hallux. Para del Sol \& Prates (1988 y 1990) lo señalado por Baxter \& Thigpen constituiría una variación anatómica en el trayecto y relaciones del nervio. Basan su discrepancia señalando que solo es posible observar esta situación en raras excepciones, esto cuando la cabeza profunda del músculo abductor del dedo mínimo no se origina del proceso medial de la tuberosidad calcánea, lo que permite que el referido ramo quede colocado entre la cara superficial de la cabeza medial del músculo cuadrado plantar.

Aunque el músculo abductor del dedo mínimo tiene dos cabezas, el nervio plantar lateral le otorga solo un ramo el que posteriormente se divide en dos, uno posterior más delgado para la cabeza profunda y otro anterior más grueso 


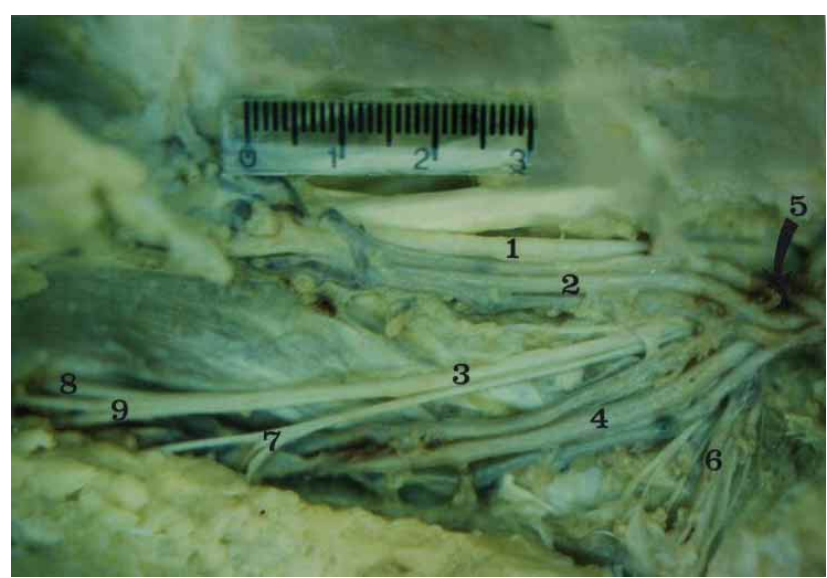

Fig. 3. Vista de la región plantar mostrando los vasos y nervios plantares. 1. Nervio plantar medial; 2. Vasos plantares mediales; 3. Nervio plantar lateral; 4. Vasos plantares laterales; 5 . Ramo para el músculo abductor del dedo mínimo; 6. Vasos que acompañan al ramo para el músculo abductor del dedo mínimo; 7. Ramo cutáneo lateral; 8. Ramo profundo del nervio plantar lateral; 9. Ramo superficial del nervio plantar lateral (Gentileza Dr. del Sol).

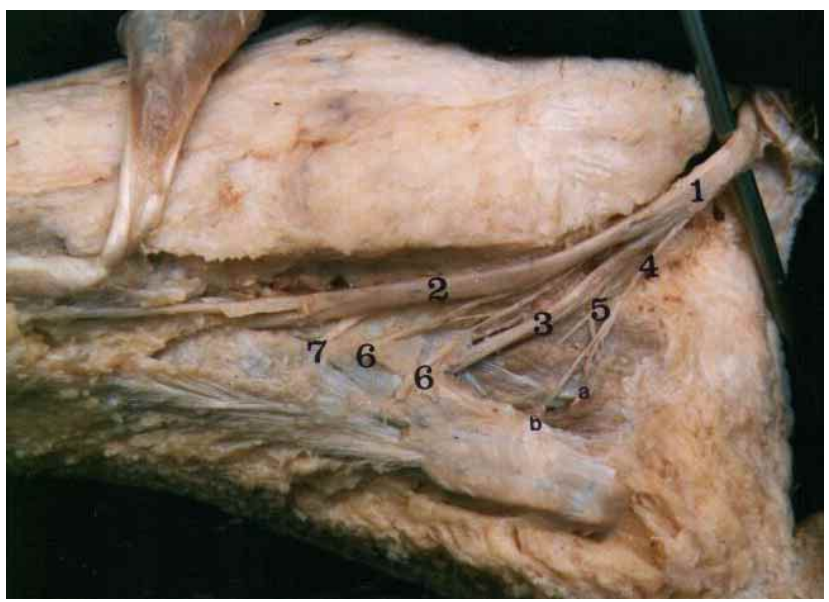

Fig. 4. Vista inferomedial del pie mostrando los nervios plantares. 1. Nervio tibial; 2. Nervio plantar medial; 3. Nervio plantar lateral; 4. Ramo para el músculo abductor del dedo mínimo con: a. Ramo posterior y b. Ramo anterior; 5. Ramo para el músculo cuadrado plantar. 6. Ramos cutáneos del nervio plantar medial; 7. Ramo para el músculo flexor corto de los dedos (Gentileza Dr. del Sol).

para la cabeza superficial. Autores clásicos como Sappey (1889) ha señalado que el ramo para el músculo abductor del dedo es siempre doble, semejante a lo relatado por Przylucki \& Jones.

Antes que el ramo nervioso alcance el margen medial de la cabeza profunda del músculo abductor del dedo mínimo, se divide en sus dos ramos menores, ocurriendo esto adjacente a la tuberosidad calcánea (del Sol \& Prates, 1988 y del Sol et al., 2002). Figs. 3 y 4. Por su parte, Przylucki \&

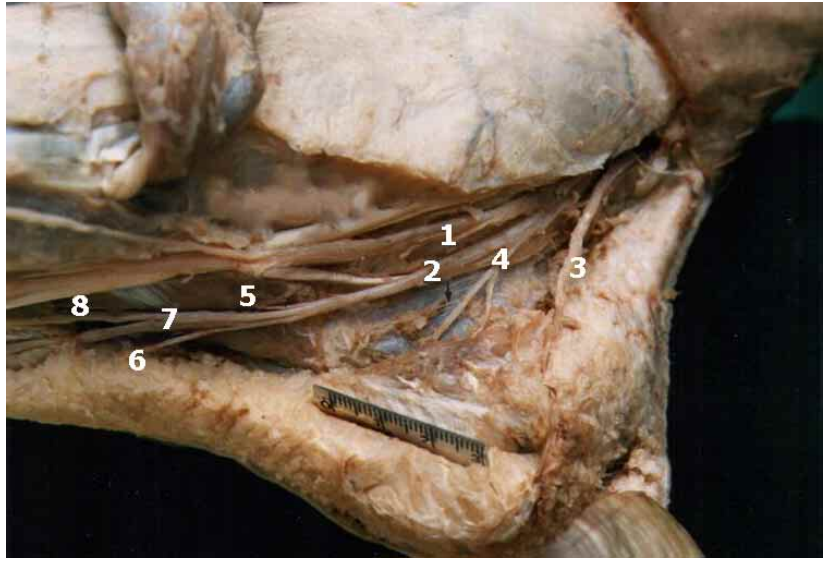

Fig. 5. Vista inferomedial del pie mostrando los nervios plantares. 1. Nervio plantar medial; 2. Nervio plantar lateral; 3. Ramo calcáneo medial; 4. Ramo para el músculo abductor del dedo mínimo con: a. Ramo posterior y b. Ramo anterior, la flecha indica un ramo vascular originándose de él; 5. Ramo para el músculo cuadrado plantar. 6. Ramo cutáneo lateral; 7. Ramo superficial del nervio plantar lateral; 8. Ramo proundo del nervio plantar lateral (Gentileza Dr. del Sol).

Jones describen la división de un ramo motor del nervio plantar lateral destinado al músculo abductor del dedo mínimo a nivel de la cabeza lateral del músculo cuadrado plantar, después de haber descansado en el ligamento plantar largo. Ambos ramos posterior y anterior, sin dividirse, ingresan a la cabeza profunda y cabeza superficial del músculo abductor del dedo mínimo, respectivamente (del Sol \& Prates, 1988). Por otra parte, Baxter \& Thigpen describieron la terminación de estos ramos en forma de abanico, antes de penetrar al músculo, situación que escapa a la normalidad de estos ramos (Figs. $1,4,6$ y 7$)$.

El ramo posterior que inerva la cabeza profunda del músculo abductor del dedo mínimo, emite antes de su terminación en las proximidades del periostio del hueso calcáneo, un ramo vascular que acompaña a los vasos destinados al músculo abductor del dedo mínimo, lo que explica la cantidad de fibras que lo componen (Fig. 6).

Del Sol et al. (2006) señalaron que el ramo sensitivo originado del ramo para el músculo abductor del dedo mínimo tenía en promedio dos fascículos, al igual que el ramo posterior del mismo nervio. Sin embargo, el ramo anterior entraba al cuerpo muscular como un solo fascículo. El número promedio de fascículos de los nervios para los músculos abductor del dedo mínimo y cuadrado plantar, esegún estos autores era de 3. El área promedio de los fascículos para el músculo abductor del dedo mínimo, era $0,165 \mathrm{~mm}^{2}$ y el número total de fibras informado fue de 1.161 (DE 111). Presley et al. a través de visualización sonográfica determinaron para este ramo un diámetro aproximado de $2 \mathrm{~mm}$, medición efctuada 


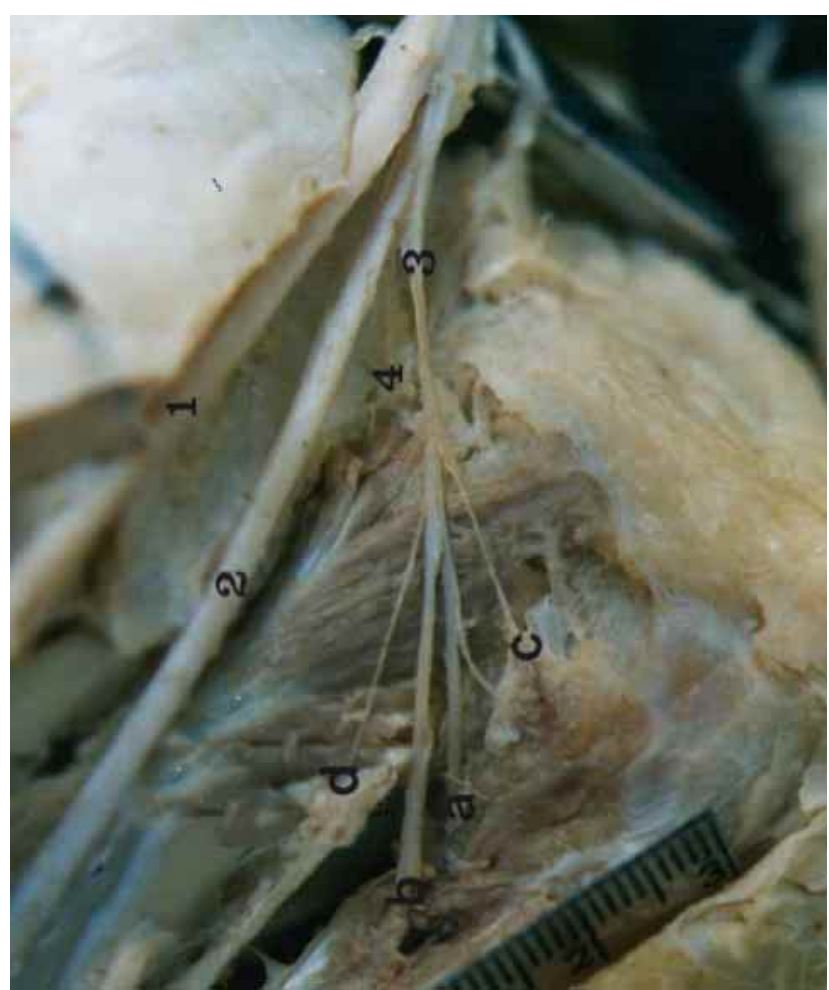

Fig. 6. Vista inferomedial de la región calcánea mostrando los nervios plantares. 1. Nervio plantar medial; 2. Nervio plantar lateral; 3. Ramo para el músculo abductor del dedo mínimo con: a. Ramo posterior. b. Ramo anterior. c. Ramo para el ligamento plantar largo y d. Ramo vascular; 4. Ramo para el músculo cuadrado plantar (Gentileza Dr. del Sol).

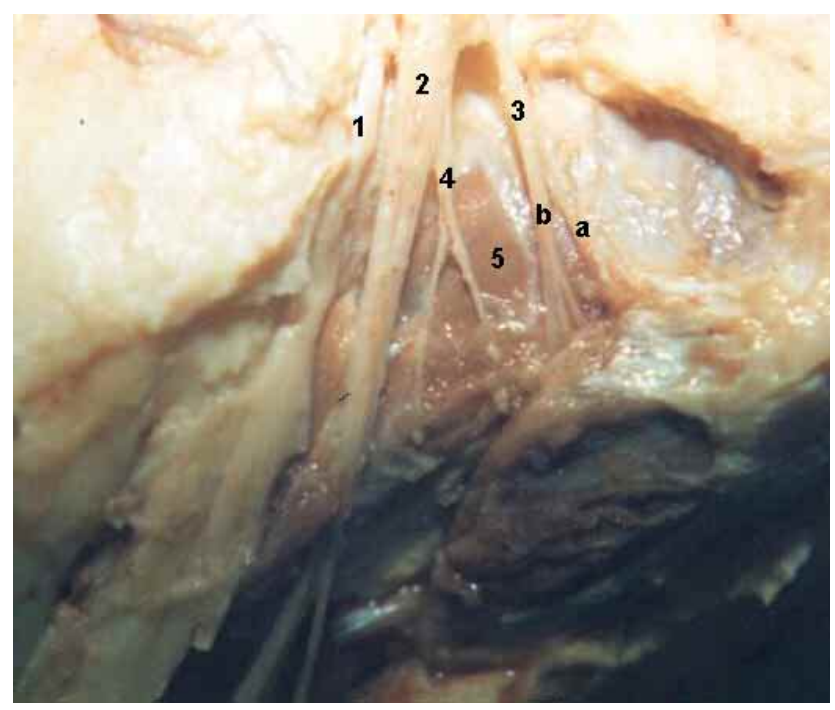

Fig. 7. Vista inferomedial de la región calcánea mostrando los nervios plantares. 1. Nervio plantar medial; 2. Nervio plantar lateral; 3. Ramo para el músculo abductor del dedo mínimo con: a. Ramo posterior. b. Ramo anterior. 4. Ramo para el músculo cuadrado plantar. (Gentileza Dr. del Sol). a nivel de su origen. Por otra parte, el promedio de fibras de cada uno de los ramos según del Sol et al. (2006) fue de 159 para el ramo sensitivo, 365 para el ramo posterior (cabeza profunda) y 518 para el ramo anterior (cabeza superficial).

Respecto del ramo para el músculo abductor del dedo mínimo, también se han efectuado estudios relacionados con dismorfía sexual en relación a la posición del nervio. Así Mizuno et al. (2015) señalaron que las posiciones de ramificación del nervio para el músculo abductor del dedo mínimo fueron superiores al eje maleolar-calcáneo en el $55 \%$ de los casos, en el eje en el $15 \%$ de los casos e inferior al eje maleolar calcáneo en el $30 \%$ de los casos. Para estos autores la ramificación del nervio estaba significativamente más próxima del eje maleolar calcáneo, en los pies de las mujeres que en el de los hombres, pero no hubo diferencias sexuales significativas en el patrón de ramificación, y en el ángulo del nervio para el músculo abductor del dedo mínimo.

Desde el punto de vista clínico el dolor subcalcáneo se puede deber a diversos factores, siendo uno de los más comunes la fascitis plantar. Sin embargo, ante la existencia de un espolón calcáneo de gran tamaño, es muy probable que éste pueda provocar un síndrome de compresión del nervio para el músculo abductor del dedo mínimo. El ramo más comprometido debiese ser el posterior el cual lleva numerosas fibras sensitivas a la región. Esta situación puede ser observado entre el $15 \%$ al $20 \%$ de los pacientes con dolor crónico de la región plantar (Baxter \& Thigpen).

En relación a los riesgos de lesiones quirúrgicas en la fasciotomía plantar percutánea, Yañez Arauz et al. (2010), en un estudio en 11 piezas cadavéricas frescas señalaron que no se evidenciaron daños en los nervios y vasos del retropié. El promedio de distancia entre el corte y el nervio plantar lateral fue de $16 \mathrm{~mm}$, y entre el corte y el nervio para el músculo abductor del dedo mínimo de 10,7 $\mathrm{mm}$. De acuerdo a lo anterior, y en contraposición con algunos otros estudios, el nervio estaría a mayor distancia de la tuberosidad calcánea, probablemente debido a variaciones anatómicas de las muestras.

Przylucki \& Jones señalaron que el nervio plantar lateral antes de su división en ramos superficial y profundo, otorga otro ramo para dicho músculo, el cual ingresa en él aproximadamente a $2 \mathrm{~cm}$ de la base del quinto metatarsiano. Creemos que es muy probable que estos autores hayan confundido este ramo, con el ramo cutáneo lateral (del Sol \& Prates, 1990). Este ramo, casi siempre constante, se origina un poco antes de la división del nervio plantar lateral, se dirige anteriormente y cruza inferiormente al músculo abductor del dedo mínimo y, algunas veces, al músculo flexor corto del dedo mínimo, termina en el tejido subcutáneo, superficial al músculo. 


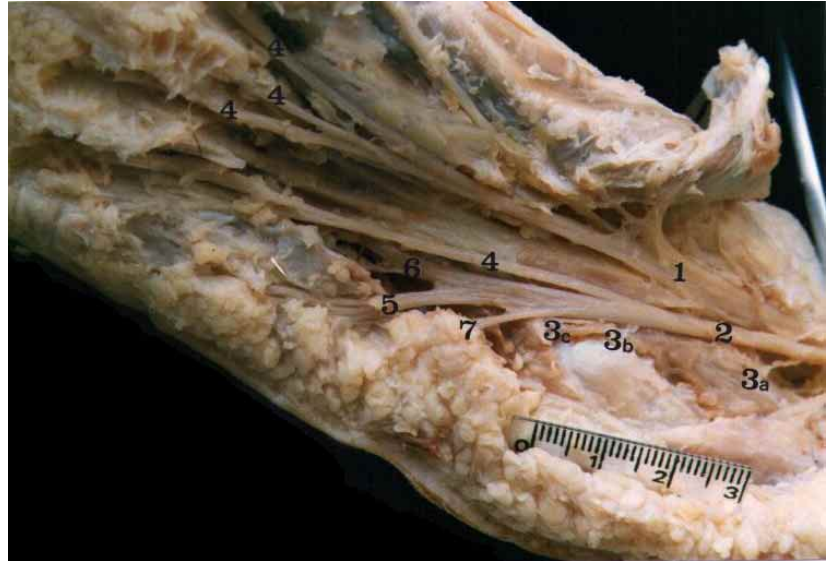

Fig. 8. Vista de la región plantar mostrando los nervios plantares. 1. Nervio plantar medial; 2. Nervio plantar lateral; 3. Ramos para el músculo cuadrado plantar con a. Ramo posterior, b. Ramo medio y c. Ramo anterior; 4. Nervios digitales plantares comunes; 5. Ramo superficial del nervio plantar lateral; Ramo profundo del nervio plantar lateral; 7. Ramo cutáneo lateral.

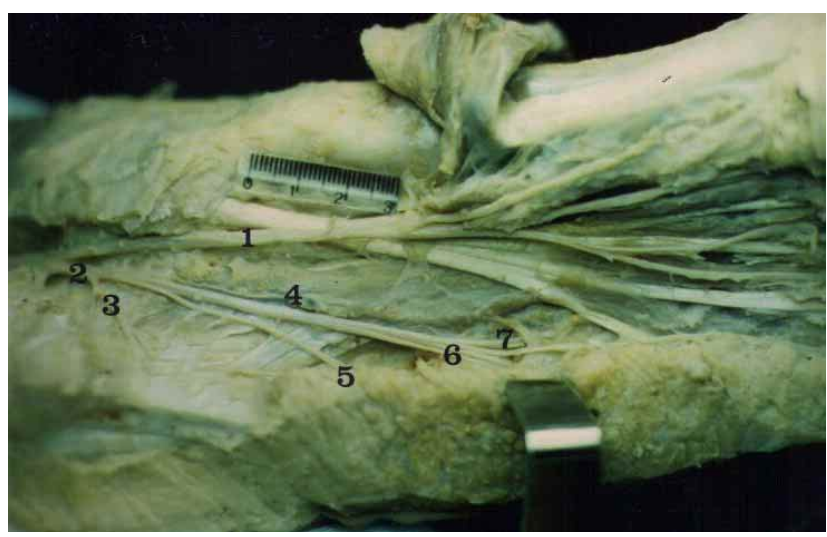

Fig. 9. Vista de la región plantar mostrando mostrando los nervios plantares. 1. Nervio plantar medial; 2. Nervio plantar lateral; 3. Ramo para el músculo abductor del dedo mínimo; 4. Ramo para el músculo cuadrado plantar; 5. Ramo cutáneo lateral; 6. Ramosuperficial del nervio plantar lateral; 7. Ramo profundo del nervio plantar lateral (Gentileza Dr. del Sol).

Una variación anatómica poco frecuente es la de un tronco común de donde se originen los ramos calcáneos mediales y el ramo para el músculo abductor del dedo mínimo. Como lo señalaron del Sol \& Prates (1988), el trayecto que siguen los ramos constitutivos de él son similares al trayecto y relaciones, que cuando se originan independientemente del nervio plantar lateral. Para Baxter \& Thigpen el origen del ramo para el músculo abductor del dedo mínimo se realiza también en conjunto con los ramos calcáneos mediales, más los dos nervios plantares, lateral y medial.

Es necesario destacar que en determinadas ocasiones se ha optado ante un dolor provocado por fascitis plantar re-

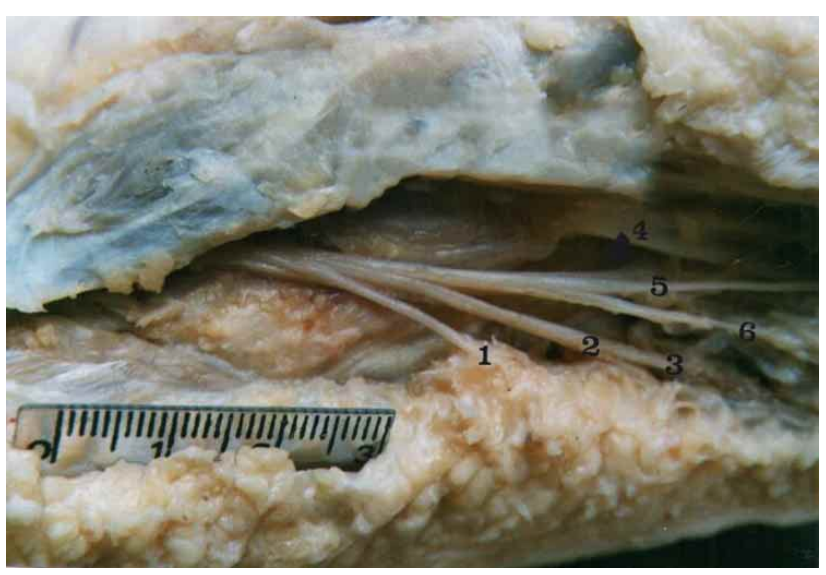

Fig. 10. Vista de la región plantar mostrando la división del nervio plantar lateral. 1. Ramo cutáneo lateral; 2. Nervio digital plantar propio del dedo mínimo; 3 . Ramo para el músculo flexor corto del dedo mínimo; 4. Ramo profundo del nervio plantar lateral (flecha); 5. Cuarto nervio digital plantar común; 6. Ramo para el tercer músculo interóseo plantar (Gentileza Dr. del Sol).

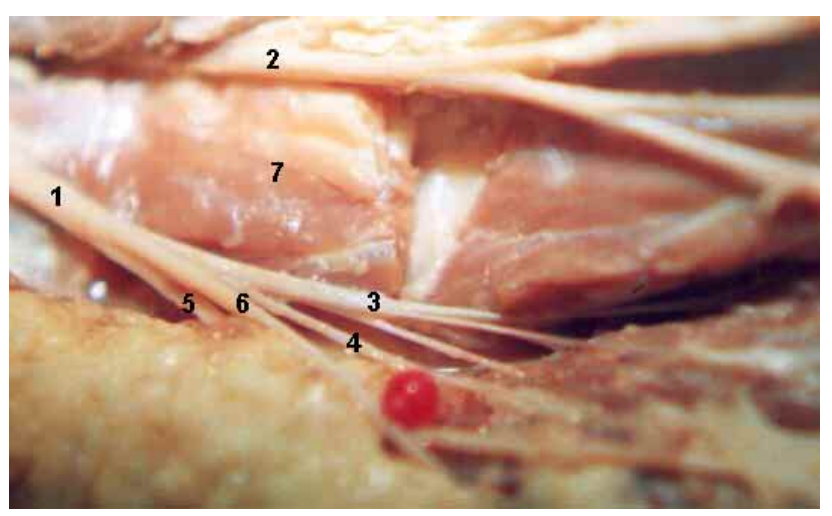

Fig. 11. Vista de la región plantar mostrando la división del nervio plantar lateral. 1. Nervio plantar lateral; 2. Nervio plantar medial; 3. Ramo profundo del nervio plantar lateral; 4. Ramo para el músculo flexor corto del dedo mínimo; 5 . Ramos cutáneo lateral; 6 . Ramo superficial del nervio plantar lateral; 7. Músculo cuadrado plantar (Gentileza Dr. del Sol).

sistente efectuar la liberación de este primer ramo del nervio plantar lateral (Sadek et al., 2015). Por otra parte, según Rodrígues et al. (2015), la atrofia grasa del músculo abductor del dedo mínimo está fuertemente asociada con aleraciones neuropáticas del ramo que inerva al músculo. Ellos también relataron una asociación significativa entre la fascitis plantar y los várices de tobillo con atrofia grado IV del músculo abductor del dedo mínimo.

Otro de los ramos musculares del nervio plantar lateral se refiere al destinado al músculo cuadrado plantar. Ninguna teoría sobre la función de este músculo, como lo señalaran Thomas \& Lyman ha sido apoyada unánimamente por 
la comunidad científica. De hecho, hay una falta de evidencia disponible para apoyar cualquier línea de pensamiento. Aunque existe controversia en cuanto al número de ramos para este músculo, pareciera ser lo más común uno o dos. Cuando es solo un ramo, éste suele dividirse en dos, uno para la cabeza medial y otro para la cabeza lateral. Del Sol \& Prates (1987) encontraron un solo ramo nervioso para el músculo cuadrado plantar en alrededor del $60 \%$ de los casos. Según estos autores el ramo para el músculo cuadrado plantar es más delgado que el ramo para el músculo abductor del dedo mínimo. Cuando el ramo es único, entra al músculo en la mayoría de los casos por la cabeza medial, próximo a la unión de las dos cabezas. Sin embargo, varios autores clásicos, entre ellos Sappey; Testut \& Latarjet, Fusari in Bertelli et al. (1913), entre otros señalan su ingreso en la cabeza lateral del músculo cuadrado plantar (Figs. 4 y 5).

Según Toye (2012) ocasionalmente el ramo destinado para el músculo abductor del dedo mínimo, le otorga también inervación al músculo flexor corto de los dedos y a la mitad lateral del músculo cuadrado plantar. No indica este autor, de donde se desprendería el ramo que inervaría la cabeza medial del músculo, dejando abierta la posibilidad a que sea procedente del nervio plantar medial, semejante a lo señalado en gran parte de la literatura clásica francesa.

Lo normal es que el ramo para el músculo cuadrado plantar se origine cubierto por la fascia del músculo abductor del hallux, pero no en contacto directo, sino separado por las numerosas anastomosis que se establecen entre las venas plantares, mediales y laterales, encontrándose además relacionado con la cara superficial de la cabeza medial del músculo cuadrado plantar (del Sol \& Prates 1987). Según estos autores este ramo acompaña al nervio plantar lateral por su lado medial en el $70 \%$ de los casos (Figs. 4, 5 y 7).

La inervación doble del músculo cuadrado plantar, por ramos procedentes de ambos nervios plantares, es decir la cabeza medial inervada por un ramo del nervio plantar medial y la cabeza lateral inervada por un ramo del nervio plantar lateral fue reportada por anatomistas franceses e italianos clásicos (Sappey, Fusari in Bertelli et al.; Rouvière (1948), Testut \& Latarjet, entre otros) (Fig. 6).

La doble inervación para el músculo cuadrado plantar siempre proviene del nervio plantar lateral y ha sido señalada por del Sol \& Prates (1987) en alrededor del $30 \%$ de los casos. Estos ramos ya habían sido reportados por Paturet (1951). El origen de los ramos se realiza tanto de la parte medial (ramo posterior para la cabeza medial) como de la parte lateral (ramo anterior para la cabeza lateral) del nervio plantar lateral. Para Bourgery el ramo que se origina de la parte medial del nervio plantar lateral es el más voluminoso.
Schroeder et al. (2014) analizaron el tipo de fibras musculares de las cabeza medial y lateral del músculo cuadrado plantar y sus resultados mostraron una homogeneidad sorprendente en la composición del tipo de fibra dentro de un individuo, con una diferencia promedio en el contenido de fibra Tipo I del 4,1 \% entre las cabezas lateral y medial. Sin embargo, entre individuos las diferencias eran muy notables. Sus resultados demostraron un alto grado de variación en las proporciones de fibras, que puede reflejar estados patológicos o fisiológicos normales como lo señalan los propios autores.

Del Sol \& Prates (1987) observaron que el ramo posterior para la cabeza medial del músculo cuadrado plantar se originaba cubierto por la fascia profunda del músculo abductor del hallux y por tanto no cruzaba el margen inferior del referido músculo. Por otra parte el ramo anterior, se originaba principalmente distal al margen inferior del músculo abductor del hallux, con lo que el nervio evita cruzar este margen grueso e inelástico mencionado por Przylucki \& Jones.

También del Sol \& Prates (1987) observaron tres ramos para el músculo cuadrado plantar. Estos autores fueron los primeros en describir detalladamente la inervación recibida por este músculo. Los ramos en la mayoría de los casos se originaban del nervio plantar lateral cubierto por la fascia de la cara profunda del músculo abductor del hallux. Okayima esquematiza entre dos ramos para el músculo cuadrado un ramo cutáneo lateral (Fig. 8).

Aún más infrecuente es el origen de un tronco común de los ramos nerviosos para los músculos abductor del dedo mínimo y cuadrado plantar desde el nervio plantar lateral, señalado por del Sol \& Prates (1988). Aparece una disposición similar en una figura presentada por Henle (1867), quien esquematiza a este ramo próximo al origen del nervio plantar lateral, el cual rodea el proceso medial de la tuberosidad calcánea y emite un ramo colateral que penetra en la cabeza profunda del músculo abductor del dedo mínimo, para enseguida dirigirse transversal a lateral y dividirse en dos ramos más pequeños, posterior y anterior, que penetran en la cabeza superficial del músculo abductor del dedo mínimo y cabeza medial del músculo cuadrado plantar, respectivamente.

El área promedio de los fascículos para el músculo cuadrado plantar según del Sol et al. (2006) es $0,070 \mathrm{~mm}^{2}$. El promedio del número de fibras según estos mismos autores para el músculo cuadrado plantar es de 419.

No existen muchos relatos, en la literatura consultada, de ramos cutáneos importantes originados del nervio plantar lateral antes de su división. Del Sol \& Prates (1990) describieron uno o dos ramos cutáneos, de mayor grosor que los 
ramos musculares. Uno de estos ramos fue denominado ramo cutáneo lateral, por ser más grueso que los otros ramos de esta parte del nervio plantar lateral y además, originarse entre los músculos flexor corto de los dedos inferiormente y cuadrado plantar superiormente (Figs. 9 y 10). Durante su trayecto este ramo cruzaba los vasos plantares laterales y el espacio comprendido entre los músculos flexor corto de los dedos y abductor del dedo mínimo, para terminar inervando el tejido subcutáneo situado inferiormente al músculo abductor del dedo mínimo y alcanzar posteriormente el margen lateral del pie. Según del Sol et al. (2006) en promedio son cuatro los fascículos que constituyen el nervio cutáneo lateral. El área promedio de estos fascículos sería de $0,041 \mathrm{~m}^{2}$

El ramo cutáneo lateral termina de manera arboriforme llegando hasta el límite del nervio sural. Este ramo según del Sol \& Prates (1990), casi en el $100 \%$ de los casos, se originaba distal al ramo para el músculo cuadrado plantar (Fig. 11). También estos autores reportaron dos ramos cutáneos laterales emitidos por el nervio plantar lateral, siendo estos infrecuentes. Estos ramos denominados cutáneos lateral posterior y anterior seguían un trayecto paralelo.

El origen directo desde el nervio plantar lateral de un ramo para el músculo flexor corto del dedo mínimo aparentemente constituye una variación (ver del Sol et al., 1990). Son escasas las descripciones anatómicas del origen y trayecto de este nervio. Frecuentemente proviene del ramo lateral del ramo superficial del nervio plantar lateral o más proximalmente del ramo superficial del nervio plantar lateral (Standring, 2010). Cabe destacar que este ramo fue atribuido a un ramo para el músculo abductor del dedo mínimo (Przylucki \& Jones).

En un estudio sobre el primer ramo del nervio plantar lateral Rondhuiss \& Huson (1986) estudiaron el curso y patrón de ramificación del nervio plantar lateral. Señalaron que durante su recorrido este primer ramo del nervio plantar lateral da dos ramos, uno de ellos penetra la inserción del músculo cuadrado plantar, envía fibras al periostio alrededor del proceso medial de la tuberosidad del calcáneo y del ligamento plantar largo y el otro inerva el músculo flexor corto de los dedos. Esta disposición y distribución no ha sido relatada por otros autores y tampoco nosotros la compartimos. La inervación del músulo flexor corto de los dedos siempre proviene de un ramo originado del nervio plantar medial.

Finalmente, se ha descrito por del Sol, en dos casos un ramo anastomótico para el nervio plantar medial, específicamente entre uno de los nervios digitales plantares comunes y un ramo del nervio plantar lateral. También se han abordado ramos nerviosos originados del nervio plantar lateral antes de su división para los ligamentos de la planta del pie, en especial para el ligamento plantar largo.
Como lo señalaron del Sol et al. (2003) el número de fascículos y de fibras nerviosas que lleve el nervio plantar lateral como también cada uno de sus ramos, va a a estar supeditado a que se mantenga el padrón de normalidad en la emisión de ramos desde su origen hasta su división. A pesar que los nervios presentan pocas variaciones en relación al sistema vascular, se hapodido observar en los diferentes estudios consultados que existe una diversidad de variaciones en los ramos otorgados por el nervio plantar lateral, especialmente en su origen. La presencia en este caso será de nervios calcáneos mediales, ramos vasculares, uno, dos o tres ramos para el músculo cuadrado plantar y más de un ramo cutáneo lateral.

Finalmente cabe señalar que en la actualidad, cada vez más se exige un conocimiento amplio y acabado de la anatomía humana regional del pie. Resulta ser particularmente importante en los procedimientos quirúrgicos, especialmente cuando esperamos que estos resultados, con abordajes menos radicales, sean los esperados.

DEL SOL, M. \& VÁSQUEZ, B. First branches of the lateral plantar nerve. Anatomo-Clinical Review. Int. J. Morphol., 35(2):765-775, 2017.

SUMMARY: The lateral plantar nerve is a terminal branch of the tibial nerve, which innervates most of the foot's musculature, and also provides sensory innervation to the sole of the foot. In this present review we address various aspects of the lateral plantar nerve from its origin to its division, emphasizing those branches that, as a result of their anatomical disposition have been identified as causing compression syndrome. Furthermore, thorough knowledge and experience of anatomical variations are essential in procedures of the plantar region. Numerous studies have been carried out to accurately describe the path and relationship of the first branches of the lateral plantar nerve. Branches originating directly from the lateral plantar nerve are for the abductor digiti minimi and quadratus plantae muscles, in addition to a lateral cutaneous branch. Among these anatomical variations it is possible to find medial calcaneal branches, common trunk for medial calcaneal branches and abductor digiti minimi muscle, vascular branches for the plantar vessels, 2 or 3 branches for the quadrate plantae muscle, common trunk for the abductor digiti minimi muscle an quadratus plantae muscle, branch for the flexor digiti minimi brevis muscle, "anastomotic" branch for the medial plantar nerve and branch for long plantar ligament. Updated and absolute knowledge of the anatomy of the foot are necessary, particularly during those surgical procedures that require precision, with fewer invasive approaches and positive results.

KEY WORDS: Lateral plantar nerve; Anatomy; Compression syndrome. 


\section{REFERENCIAS BIBLIOGRÁFICAS}

Baxter, D. E. Thigpen, C. M. Heel pain - operative results. Foot \& Ankle, 5:16-25, 1984.

Belen, J. Orthodromic sensory nerve conduction of the medial and lateral plantar nerves. Am. J. Phys. Med., 64:17-23, 1985.

Bordelon, R. L. Subcalcaneal pain. Clin. Orthop., 177:49-53, 1983.

Bourgey, J. M. Traité complet de l'anatomie de l'homme. Paris, Delaunay Éditeur, 1844. V. 3.

Davis, T. J. \& Schon, L. C. Branches of the tibial nerve. Anatomic variations. Foot \& Ankle Int. 16(1):21-9, 1995.

Dellon, A. L. \& Mackinnon, S. E. Tibial nerve branching in the tarsal tunnel. Arch. Neurol., 41:645-6, 1984.

Del Sol, M.; Binvignat, O.; Prates, J. C. Ribeiro, E. Inervação do músculo flexor curto do dedo mínimo do pé no homem. Folha Méd. (BR), 100(3):49-52, 1990.

Del Sol, M.; Olave, E.; Gabrielli, C. \& Mandiola, E. División de la arteria tibial posterior. Rev. Chil. Anat., 17(1):99-102, 1999.

Del Sol, M.; Olave, E.; Gabrielli, C.; Mandiola, E. \& Prates, Innervation of the abductor digiti minimi muscle of the human foot. Anatomical basis of the entrapment of the abductor digiti minimi nerve. Surg. radiol. Anat., 24:18-22, 2002.

Del Sol, M. \& Prates, J. C. Inervação do músculo abductor digiti minimi do pé no homem. Folha Méd. (BR), 96(4):209-13, 1988.

Del Sol, M. \& Prates, J. C. Inervação do músculo quadrado plantar no homem. Rev. Bras. Ciên. Morfol., 4(1):20-3, 1987.

Del Sol, M. \& Prates, J. C. Ramos cutâneos do nervo plantar lateral antes de sua divisão em ramos superficial e profundo (Ramo cutâneo lateral). Rev. IATROS, 6:63-5, 1990.

Del Sol, M. \& Prates, J. C. Anatomia do músculo abdutor do dedo mínimo do pé no homem. Rev. Bras. Cir., 80(6):371-5, 1990.

Del Sol, M.; Prates, J. C.; Olave, E.; Mandiola, E. \& Gabrielli, C. Relaciones anatómicas entre los nervios tibial y plantares y los vasos tibiales posteriores a nivel del retináculo de los músculos flexores. Rev. Chil. Anat., 13(2):195-202, 1995.

Del Sol, M.; Prates, J. C.; Olave, E. \& Ribeiro, E. Contribución al estudio de los ramos calcáneos mediales originados del nervio plantar lateral. F. Méd., 108(1-2):35-8, 1994.

Del Sol, M.; Prates, J. C.; Olave, E. \& Ribeiro, E. Medial calcaneal branches from the lateral plantar nerve. Rev. Chil. Anat., 10(2):133-8, 1992a.

Del Sol, M.; Prates, J. C. \& Ribeiro, E. Nível de divisão do nervo tibial. Folha Méd. (BR). 102(5):151-4, 1991.

Del Sol, M.; Vásquez, B. Nicklas, C. Morfometría de los ramos del nervio plantar lateral antes de su división en ramos superficial y profundo. Int. J. Morphol., 24(2):191-194, 2006.

Fusari, R. Neurologia. In Bertelli, D.; Fusari, R.; Romiti, G.; Sala, L.; Valenti, G. \& Versari, R. Trattato di anatomia umana. Milano, Casa Editrice Dottor Francesco Callardi, 1913. V. 4.

Henle, J. Handbuch der systematishen Anatomie des Menschen. Braunshweig, ruck und Verlag von Friedrich Vieweg und Sohn, 1867.

Henricson, A. S. \& Westlin, N. E. Chronic calcaneal pain in athletes: entrapment of the calcaneal nerve? Am. J. Sports Med., 12:152-4, 1984.

Kopell, H. P. \& Thompson, W. A. L. Peipheral entrapment neuropathies of the lower extremity. N. Engl. J. Med., 262:56-60, 1960.

Louisia, S. \& Masquelet, A. C. The medial and inferior calcaneal nerves: an anatomic study. Surg. Radiol. Anat., 21(3):169-73, 1999.

Lumsden, D. B.; Schon, L. C.; Easley, M. E.; Douiguih, W. A.; Anderson, C. D.; Miller, S. D. \& Ottey, D. K. Topography of the distal tibial nerve and its branches. Foot \& Ankle, 24(9):696-700, 2003.

Martín-Oliva, X.; Elgueta-Grillo, J.; Veliz-Ayta, P.; Orosco-Villaseñor, S.; Elgueta-Grillo, M. \& Viladot-Perice, R. Variantes anatómicas del nervio calcáneo medial y nervio de Baxter en el túnel del tarso. Acta ortop. Mex., 27(1):38-42, 2013.

Mestdagh, H.; Houcke, M.; Moulront, S. \& Laraki, A. Contribution à l'étude de l'innervation sensitive de la coque talonnière. Acta Anat., 108:12431, 1980.

Mizuno, D.; Naito, M.; Hayashi, S.; Ohmichi, Y.; Ohmichi, M. \& Nakano, T. J. Foot \& Ankle Res., 8:22, 2015.

Paturet, G. Traité d'Anatomie Humaine. Paris, Masson, 1951. V. 3.

Presley, J. C.; Maida, E.; Pawlina, W.; Murthy, L.; Ryssman, D. B. \& Smith, J. Sonographic visualization of the first branch of the lateral plantar nerve (Baxer nerve). Technique and validation using perineural injections in a cadaverc model. J. Ultrasound Med, 32:1643-52, 2013.

Przylucki, H. \& Jones, C. L. Entrapment neuropathy of muscle branch of lateral plantar nerve. J. Am. Podiatry Assoc., 71:119-24, 1981.

Ribet, R. M. Anatomie schématique de l'appareil nerveux. Les nerfs rachidiens. Paris, Octave Doin, 1953.

Rodrigues, R. N.; Lopes, A. A.; Torres, J. M.; Mundim, M. F.; Silva, L. L. G. \& Carvalho e Silva, B. R. Compressive neurophaty of the first branch of the lateral plantar nerve: a study by magnetic resonance imaging. Radiol. Bras., 48(6):368-72, 2015.

Rondhuis, J. J. \& Huson, A. The first branch of the lateral plantar nerve and heel pain. Acta Morphol. Neerl. Scand., 24(4):269-79, 1986.

Rouvière, H. Anatomie Humaine. 6 èd. Paris, Masson, 1984. V. 3.

Sadek, A. F.; Fouly, E. H.; Elian, M. M. Lateral plantar nerve release with or without calcaneal drilling for resistant plantar fasciitis. J. Orthopaedic Surg., 23(2):237-40, 2015.

Sappey, PH. C. Traité d'anatomie descriptive. 4. èd. Paris, Lecrosnier et Babé, 1889. V. 3.

Schroeder, K. L.; Rosser, B. W. C. \& Kim, S. Y. Fiber type composition of the human quadratus plantae muscle: a comparison of the lateral and medial heads. J. Foot Ankle Res., 7:54, 2014.

Soibelman, J. Observações anatômicas sôbre os rami calcanei mediales do nervo tibial no homem. Tese de Doutorado, Escola Paulista de Medicina, São Paulo, 1984.

Standring, S. Gray's Anatomia. A base anatômica da prática clínica. 40 ed. Elsevier, Rio de Janeiro, 2010.

Tandler, J. Tratado de anatomía sistemática. Barcelona, Salvat, 1933. V. 4.

Tanz, S. S. Heel pain. Clin Orthop., 28:69-78, 1963.

Testut, L. \& Latarjet, A. Tratado de anatomía humana. Barcelona, Salvat, 1969.

Thomas, P. \& Lyman, B. S. A review of the function of the quadratus plantae. Foot Ankle Online J., 2(11):5, 2009.

Torres, G. A. L. \& Ferreira, C. M.Estudo da anatomia do nervo tibial e sesu ramos ao nível do terço distal da perna. Acta ortop. bras., 20(3):15764, 2013.

Toye, L. Baxter's nerve (first branch of the lateral plantar nerve) impingement. MRI Web Clinic, 2012.

Ulcay, T.; Uzun, A. \& Ziylan, T. The origin and branching of medial calcaneal nerve in newborn foetuses. J. Anat. Soc. India, 63 Supp. 2:S1S5, 2014.

Woodburne, R. T. Anatomía Humana. 6 ed. Rio de Janeiro, Guanabara \& Koogan, 1984.

Yañez Arauz, J. M.; del Vecchio, J. J.; Raimondi, N. \& Codesido, M. A. Riesgo de lesiones quirúrgicas en la fasciotomía plantar percutánea: Estudio anatómico en cadáveres frescos. Rev. Asoc. Argent. Ortop. Traumatol., 76(2):141-5, 2011.

\section{Dirección para correspondencia: \\ Dra. Bélgica Vásquez P. \\ Facultad de Ciencias de la Salud \\ Universidad de Tarapacá \\ Arica - CHILE}

Email: bvasquezp@uta.cl

Recibido : 22-12-2016

Aceptado: 18-02-2017 